\title{
Analysis of Inertial Choice Behaviour based Expected and Experienced Savings from a Real-World Route Choice Experiment
}

\author{
Jaap Vreeswijk ${ }^{1}$, Hesham Rakha ${ }^{2}$, Eric van Berkum ${ }^{3}$ and Bart van Arem ${ }^{4}$ \\ ${ }^{1}$ University of Twente \\ ${ }^{2}$ Virginia Tech \\ ${ }^{3}$ University of Twente \\ ${ }^{4}$ Delft University of Technology \\ 1j.d.vreeswijk@utwente.nl, ${ }^{2}$ hrakha@vt.edu, ${ }^{3}$ e.c.vanberkum@utwente.nl, \\ ${ }^{4}$ b.vanarem@tudelft.nl
}

\begin{abstract}
In the context of route choice, inertial choice behaviour shows that drivers make choices that are satisfactory rather than optimal. Consequently, drivers may not necessarily respond to changes in the traffic system. Instead it is assumed that drivers only alter their choice when the utility difference between alternatives becomes larger than some individualsituation-specific threshold. Route choice data from a real-world driving experiment was used to study inertial behaviour and to estimate inertia thresholds. The data analysis consisted of two parts. One defined as expected travel time savings relative to choice alternatives and another defined as experienced travel time savings on the current route. With regard to the former it was found that on average roughly one-quarter of the choices were inertial choices. Small travel time differences and dominant non-travel time route attributes had a positive effect on the frequency of inertial choices. Based on lost travel time (compared to shortest time) for routes with an average travel time of 8 to 16 minutes, inertia thresholds up to 2.90 minutes or $33 \%$ of the average travel time were found, while thresholds up to 1.22 minutes or 13\% were most common. Considerable differences between OD-pairs and routes indicated that thresholds are probabilistic and dependent on the choice set. Considering experienced savings, it was found that on average roughly half of the choices concerned inertial behaviour. Inertia thresholds up to 3.77 minutes or $47 \%$ of the average travel time were found, while thresholds up to 0.97 minutes or $11 \%$ were more common. Switching behaviour could not be explained by experienced savings as participants were much less responsive to experienced loss on the current route as opposed to expected loss relative to the choice alternative.
\end{abstract}

Keywords: No Keyword

\section{Introduction}

In the 1970's and again more recently, behavioural economics is rapidly gaining ground both outside and inside the transport research arena. Behavioural economics draws on the aspects of (cognitive) psychology, social sciences and economics, and studies the motives and behaviours that explain deviations from rational behaviour [1]. In contrast to the rational economic model, human rationality is about our distance from perfection given the observation that people have limited knowledge and constrained cognitive abilities, leading to prejudiced reasoning, biased perception and a type of behaviour that is characterised by repeatedly choosing suboptimal routes from the perspective of travel time minimisation. A generic term for this kind of behaviour is 'bounded rationality' that was first introduced by Herbert Simon [2]. 
From the 1970's onwards, many researchers showed that boundedly rational behaviours are neither random nor senseless, but that they are systematic, consistent, repetitive, and therefore predictable. Most distinguished and frequently cited are works of Kahnemann and Tversky [for an overview see 3], and McFadden [e.g., 4]. Moreover, it is widely acknowledged that these behaviours have important implications for example on prediction capabilities of choice models and the effectiveness of transport policy. For example, a relevant notion in the context of boundedly rational behaviour relates to route switching behaviour and is that drivers supposedly only alter their behaviour or choice when the utility difference in the transport systems or their trip, becomes larger than some individualspecific threshold [5]. Like boundedly rationality, the term 'threshold' is relatively generic. It acknowledges the potential existence of limits, boundaries or cut-offs of perception and consideration of attributes by an individual, as a result of some behavioural mechanism [6].

The notion of indifference bands and thresholds is well-known in economics and marketing as it is closely related to price elasticity. As a complement to price elasticity, thresholds add the condition that consumers do not respond to small price changes within a 'pricing indifference bands', i.e. a range of possible prices up to which price changes have little or no impact on customer purchase decisions [7]. It is argued that this 'range of inattention' along the demand curve gives retailers an incentive for small price increments to increase profit [8]. Analogically and as conceptualised and demonstrated in earlier work by the authors $[9,10]$, when applied in the context of transport thresholds may give policy makers, road operators and traffic engineers an incentive 'lever' to modify choice attributes, which in turn will increase the performance of the traffic system.

Unmistakably, the challenge and at the same time objective of this paper is to collect empirical evidence and estimate the indifference band threshold. Remarkably, the literature on empirical research is limited despite the numerous requests for empirical evidence to validate theories derived from behavioural economics and to develop better descriptive models for travel choice behaviour. The next Section further describes the background of this research, followed by the research objective and approach. Next the setup of the realworld driving experiment is described to demonstrate how the route choice data were collected and used. Thereafter the analysis results are presented. The final Section presents the conclusions of the study and outlines future research activities.

\subsection{Background}

An important limitation with regard to bounded rationality and thresholds is that underlying behavioural and cognitive mechanisms may differ in a strict sense, but that it is impossible to distinguish between them based on observed choice alone. Therefore it is important to clearly hypothesise studied behaviours and outline the assumptions that are adopted. To further specify the concepts of bounded rationality and thresholds, the work of Cantillo and Ortuzar [6] is used as a starting point to distinguish three perspectives.

Habit forms when automated cognitive processes take control as the decision maker repeatedly choses the same alternative [11]. Moreover, if the cost of searching for and constructing new alternatives is too high, or if it has too much associated risk, people make an effort-accuracy trade-off and will tend to reuse past solutions that make behaviour easier and less risky [12]. Van Berkum and Van der Mede [13] defined habit as the propensity to repeat certain behaviours, not necessarily preceded by change or a changing force. Although similar to habit, inertia also acknowledges that the mere action of choosing a particular alternative makes it more probable that the alternative is chosen again on the next day, it adds to this notion that the reason for this repetition may in fact be that the anticipated (expected) quality of an alternative is much higher than that of its competitors [14]. As it were, the so-called inertial effect increases the utility of the current path which means that the same observed behaviour can still prevail after a change [15].

The notion of a minimum perceptible change implies that attribute changes below the threshold do not cause a reaction in the individual (as perceptually the utilities do not 
change). Theories on 'just noticeable differences' suggest that people may be unable to perceive small differences in the price of products [16], or just not interested in these differences as it does not pay off to notice them [8]. This phenomenon is complex since changes can accumulate and eventually exceed the threshold; while in parallel there might be adjustments in individual behaviour, dependent on the speed of change, which in turn can modify the threshold. Closely related are studies that deal with limited awareness which primarily focus on the discovery of (new) utility differences. Generally, awareness may either result from direct experience (i.e., by having chosen the alternative on one of the preceding days) or from indirectly noticing the change (e.g., from a friend or through a travel information service) [17].

Although it is more common practice to assume that individuals make trade-offs among attributes [12], people may actually behave in a non-compensatory manner as in the elimination-by-aspects (EBA) model of [18] or the satisficing heuristic [2]. Based on personal preferences, individuals are assumed to have both a ranking of attributes and minimum acceptable thresholds for each of the attributes. The process begins with the most important attribute, the threshold of which is retrieved and all alternatives with attribute values over the threshold are eliminated. The process is repeated for the remaining attributes in order of importance until one alternative satisfies them all. If none or more than one alternative satisfies all the threshold constraints the preferred one may be selected in a compensatory manner. On top of this process, satisficing behaviour states that decision makers will continue using the chosen alternative as long as it 'suffices' in 'satisfying' (together satisficing) the decision maker's goals. Moreover, it is assumed that the decision maker will not continue his/her search for a more optimal alternative. Based on the principle of EBA and satisficing, some analysis have shifted their attention from the decision making process to the choice set formation process as pruning of rejected alternatives may improve model predications considerably $[19,20]$.

The different perspectives are very closely related and should be considered complements rather than substitutes, as for example inertia and awareness limitations may exist simultaneously [17]. Yet, the empirical identification of such an integrated model is not trivial. For example, it is impossible to derive, from the observation that a traveller repeatedly chooses one particular route that performs less than another available route, whether this results from individual preferences (rejection of alternatives), satisficing behaviour, limited awareness, perceptual errors, habit or inertia.

\subsection{Objective and Approach}

Earlier research of the authors addressed the topic of indifference band thresholds by studying the influence of perception bias on route choice, and how route attributes and past decisions affect perception [21,22]. To complement this research, the aim of this paper is to observe the frequency of different choice strategies, to determine how they are influenced by situational variables and to quantify the thresholds associated to the strategies.

From the perspective of indifference band thresholds the main interest can be summarised as the inertial effect i.e., a traveller repeatedly chooses one particular route that performs less than another available route. It is important to realise that in the context of decisionmaking, the inertial effect may have different meaning. For example, in case of inertia or reluctance to change, travellers are supposed to be open to multiple choice alternatives but to be indifferent to differences in the utility of these alternatives. On the other hand, in case of satisficing behaviour, travellers are assumed to strictly focus only on the utility of the chosen alternative and to be indifferent to decreases in the utility. In this paper we refer to the extent travellers are indifferent to either type of disutility as the inertia threshold. 


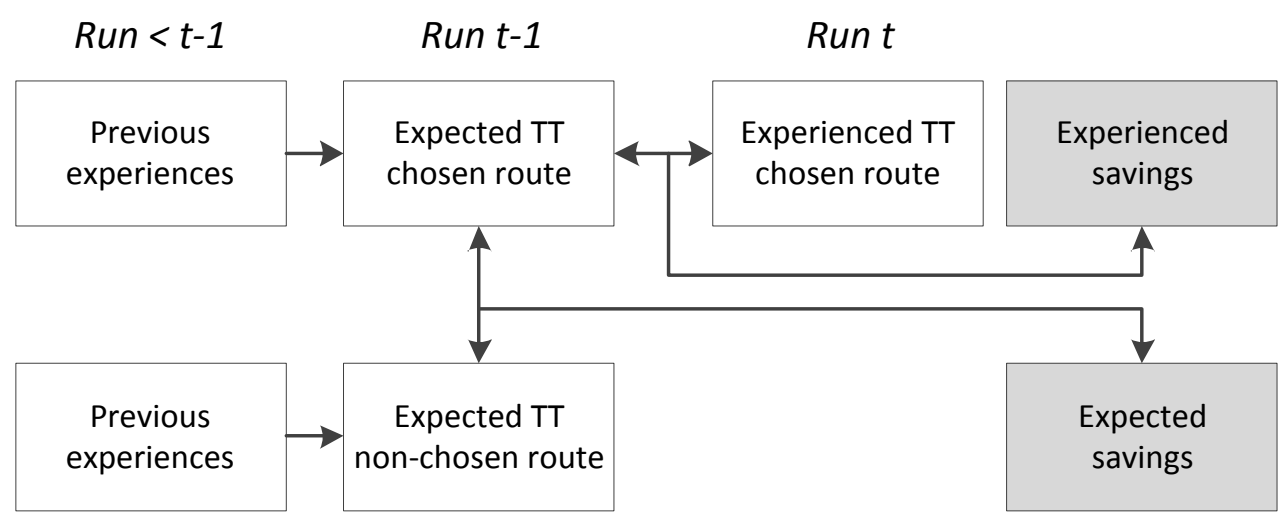

\section{Figure 1. Logic Expected Savings and Experienced Savings}

The data analysis in this paper will focus on route switching behaviour and inertial choices in particular. In anticipation to the two readings of the inertial effect we introduced expected savings relative to choice alternatives and experienced savings on the current route. The former is aligned to mechanisms of inertia and reluctance to change, whereas the latter is aligned to mechanisms of satisficing behaviour. Their computation logic is also shown in Figure 1. In this paper expected savings are defined as the difference between the expected travel times of two routes after run $\mathrm{t}-1$, assuming the driver will repeat the current choice at run $\mathrm{t}+1$. A positive value indicates a gain (i.e., travel time of the current choice < travel time choice alternative) whereas a negative value indicates a loss. Similarly, experienced savings are defined as the difference between the experienced travel time of the current route for run $\mathrm{t}$ and the expected travel time of that route after run $\mathrm{t}-1$. A positive value indicates a gain (i.e., experienced travel time < expected travel time) whereas a negative value indicates a loss. Note that for comparison of expected travel times, we assumed that participants should have chosen both alternatives at least once.

Expected and experienced savings were calculated using day-to-day route choice data from a real-world driving experiment [23]. This data and the data treatment are further described in the next Section. Based on this data, combined with data from a post-task questionnaire, Tawfik and Rakha [23, 24] had already found that: 1) perception were about $60 \%$ accurate and drivers' perception of travel speeds were more accurate than their perceptions of travel time; 2) the larger the difference between the two alternative routes is (i.e., the more salient the signal), the more accurate are the driver perceptions; 3 ) there are significant discrepancies between stochastic user equilibrium and real-world route choices which can best be explained by travel time perceptions, travel time reliability and small differences between the travel times of alternatives, and 4) Markov process updating of experienced travel times was not different from the average-based calculations which shows that the latest route experience was not dominant. It is important to note that Tawfik and Rakha assessed driver experiences, perceptions and choices mainly on an aggregate level and that their results to a large extend are based on data from the post-task questionnaire. The analysis presented in this paper extends this work by studying the revealed preference data in more detail and only using the route choice data.

\section{Data Description}

\subsection{Setup Route Choice Experiment}

Data was collected through a real real-world route choice experiment in Blacksburg, Virginia in the United States [23]. A total of 20 participants were involved in this study. Each participant was asked to complete 20 experimental runs over 20 days during regular school week days of the academic spring semester of 2011. Experimental runs were 
scheduled only during one of three traffic peak hours: morning (7-8 am), noon (12-1 pm), and evening (5-6 pm). It should be noted that the 20 runs for a driver were done at the same time each day. All participants were given the same five Google Map print outs, each map representing one OD-pair and two alternative routes. For each experimental run, participants were asked to drive a research vehicle and make these five OD-pairs assuming that the provided alternative routes were the only routes available between the points of origin and destination. The OD-pairs and the alternative routes were selected to ensure differences in the five choice situations. All driver choices as well as the experienced travel conditions were recorded via a GPS unit placed on board of the vehicle and a research escort that always accompanied the participants. Participants were instructed to behave in the same manner they behave in real life.

\subsection{Route Characteristics}

Table 1 shows the properties of the 10 routes on the 5 OD-pairs. Travel time difference denotes the average travel time difference between the two routes. Based on Monte Carlo simulation by Tawfik and Rakha (2012), the probability of the odd route being shorter in time and quicker in speed than the even route was determined. The OD-pairs can be characterised as follows:

- OD-pair 1: both routes have almost equal travel times, but route 2 has a slightly higher average speed.

- OD-pair 2: route 3 is the shorter time route, but in terms of average speed route 4 is clearly the better alternative.

- OD-pair 3: route 5 outperforms route 6 in both travel time and average speed. However, route 5 is a route with high traffic volumes.

- OD-pair 4: route 8 is a little shorter in time and has a higher average speed. However, route 8 passes through the school campus and there is risk being caught in campus traffic.

- OD-pair 5: route 10 is clearly the shorter time route while the average speed of both routes is nearly equal.

Table 1. Route Characteristics and Choice

\begin{tabular}{|c|c|c|c|c|c|c|c|c|}
\hline $\begin{array}{l}\text { OD } \\
\text { pair }\end{array}$ & Route & \begin{tabular}{|c|} 
Average \\
TT (min.)
\end{tabular} & $\begin{array}{l}\text { Travel Time } \\
\text { diff. (min.) }\end{array}$ & $\begin{array}{c}\text { Probability } \\
\mathbf{T T}_{\text {odd }}<\mathbf{T T}_{\text {even }}\end{array}$ & $\begin{array}{c}\text { Average Travel } \\
\text { Speed }(\mathbf{k m} / \mathbf{h})\end{array}$ & $\begin{array}{l}\text { Probability } \\
\text { TS }_{\text {odd }}>\text { TS }_{\text {even }}\end{array}$ & $\begin{array}{c}\# \\
\text { choices }\end{array}$ & $\begin{array}{c}\% \\
\text { choices }\end{array}$ \\
\hline \multirow{2}{*}{1} & 1 & 8.5 & 0.1 & \multirow{2}{*}{$48.3 \%$} & 36.4 & \multirow{2}{*}{$26.6 \%$} & 136 & $34 \%$ \\
\hline & 2 & 8.4 & $1 \%$ & & 43.3 & & 264 & $66 \%$ \\
\hline \multirow[b]{2}{*}{2} & 3 & 15.2 & 1.5 & \multirow{2}{*}{$78.5 \%$} & 42.6 & \multirow[b]{2}{*}{$0.1 \%$} & 254 & $63 \%$ \\
\hline & 4 & $\begin{array}{l}16.7 \\
16.7\end{array}$ & $9-10 \%$ & & 63.2 & & 146 & $37 \%$ \\
\hline \multirow[b]{2}{*}{3} & 5 & 7.7 & 1.6 & \multirow{2}{*}{$85.4 \%$} & 44.5 & \multirow{2}{*}{$91.8 \%$} & 275 & $69 \%$ \\
\hline & 6 & 9.3 & $17-21 \%$ & & 37.8 & & 125 & $31 \%$ \\
\hline \multirow{2}{*}{4} & 7 & 10.2 & 0.6 & \multirow{2}{*}{$35.2 \%$} & 29.5 & \multirow{2}{*}{$0.2 \%$} & 75 & $19 \%$ \\
\hline & 8 & 9.6 & $6 \%$ & & 48.2 & & 325 & $81 \%$ \\
\hline \multirow{2}{*}{5} & 9 & 10.5 & 2.5 & \multirow{2}{*}{$5.0 \%$} & 33.3 & \multirow{2}{*}{$40.0 \%$} & 39 & $10 \%$ \\
\hline & 10 & 8.0 & $24-31 \%$ & & 34.0 & & 361 & $90 \%$ \\
\hline
\end{tabular}

Table also shows the choices of the participants which reveal that routes 2, 3 and 5 but specially routes 8 and 10 were the preferred routes. Overall, the choice data shows that in $74 \%$ of the cases the average shortest time route was chosen. However, between OD-pairs there are some clear differences, e.g., 63\% for OD-pair 2 and 90\% for OD-pair 5. Especially for some OD-pairs there is a considerable amount of choices that cannot be explained by travel time alone. For a better understanding of these choices the next Sections will examine the data in more detail. 


\subsection{Data Treatment - Expected and Experienced Savings}

For each driver and each run the data provides the selected route and the experienced travel time, as is illustrated in Figure. From this data it is easy to identify the runs in which a driver switched from one route to the other (indicated by the black dots).

The average experienced travel time (i.e., expected travel time) was calculated after each run. According to literature, there exist different ways drivers integrate past experiences. One approach is to integrate past experienced cost according to a weighted average [e.g., 25], for example by assigning equal weights to all past experiences or by assigning weights through exponential smoothing [e.g., 26]. Under the myopic adjustment rule, the perceived travel time is a function of the latest day's outcome exclusively [e.g., 27]. The third approach considers perceived travel time as a weighted average of the historically perceived travel time and the time provided by advanced traveller information systems [e.g., 28]. However, earlier work by Tawfik and Rakha [24] on this data set showed that a Markov process updating of experienced travel times was not different from the average-based calculations. For this reason expected travel times were calculated as a straight average over all past experienced travel times (see Figure 2).

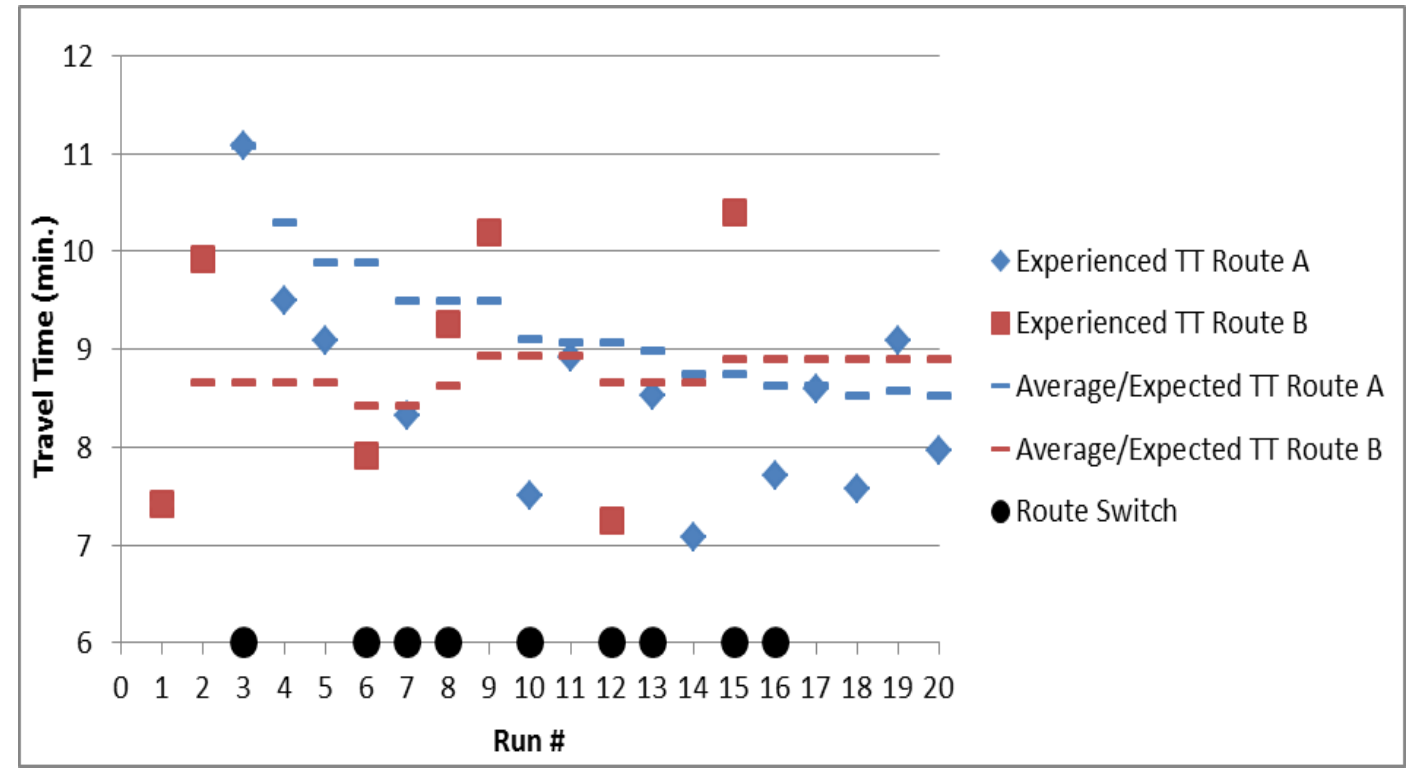

Figure 2. Example Route Choice Data of 1 Individual on 1 OD-pair

Note that the assumption that participants should have chosen both alternatives at least once reduced the sample size from 2000 to 1293 . This is mainly caused by participants who used the same route for the entire 20 runs as is described in the next Section. The interquartile range of expected savings is $-0.46-1.69$ minutes and a median of 0.53 minutes, while the interquartile range of experienced savings is $-0.70-2.00$ minutes and a median of 0.70 minutes.

\subsection{Limitations}

First of all, since data was collected in normal driving conditions on public roads, the experimenters had no control over the traffic conditions and actual travel times while the experienced travel times were dependent on the subsequent choices of the participants. In addition, run by run contextual data that might explain route choice behaviour was not available. For these reasons, the choice situations as revealed in the data (e.g., the average experienced travel times of two routes) were not uniformly balanced but naturalistic. 
Secondly, perception data directly obtained from the participants is only available from the pre-task and post-task questionnaires. That is, the data were obtained before and after the 20 runs and therefore are fairly aggregated. As run by run perception data is not available, experienced travel times were used to calculate expected travel times. Note that assuming that these represent the perception of the participants implies that perfectly rational perception is assumed. This is ambiguous and in contrast with earlier statements on perception bias. However, contrasting the experienced and expected travel times with the actual choices of the participants allowed to evaluate the rationality of these choices. In reverse it allowed to derive conclusions on the accuracy of 'rational' perceptions and as such perception thresholds.

Thirdly, nearly all participants indicated in the pre-task questionnaire that they were moderate-to-very familiar with all choice alternatives. This implies that respondents already had pre-knowledge about local traffic conditions, but not necessarily about the choice situations. Findings on learning and choice patterns should be interpreted bearing this in mind.

Finally, the five routes completed by the respondents were actually part of a trip chain. Although the participants were thoroughly instructed to behave as naturalistic as possible, the realism and importance of the factor of arrival time can be questioned.

\section{Overall Results}

\subsection{Behaviour Types and Switching}

Based on the pattern of the route choice data as presented in Figure (all 2000 samples) it is possible to distinguish four Driver Behaviour Types (DBT) as first introduced by Tawfik and Rakha [23]. The four DBT and their frequency are shown in Figure 3: Stayers, Tryers, Explorers and Switchers. New compared to the work of Tawfik and Rakha is the disaggregation to the level of OD-pairs which offers additional valuable information.

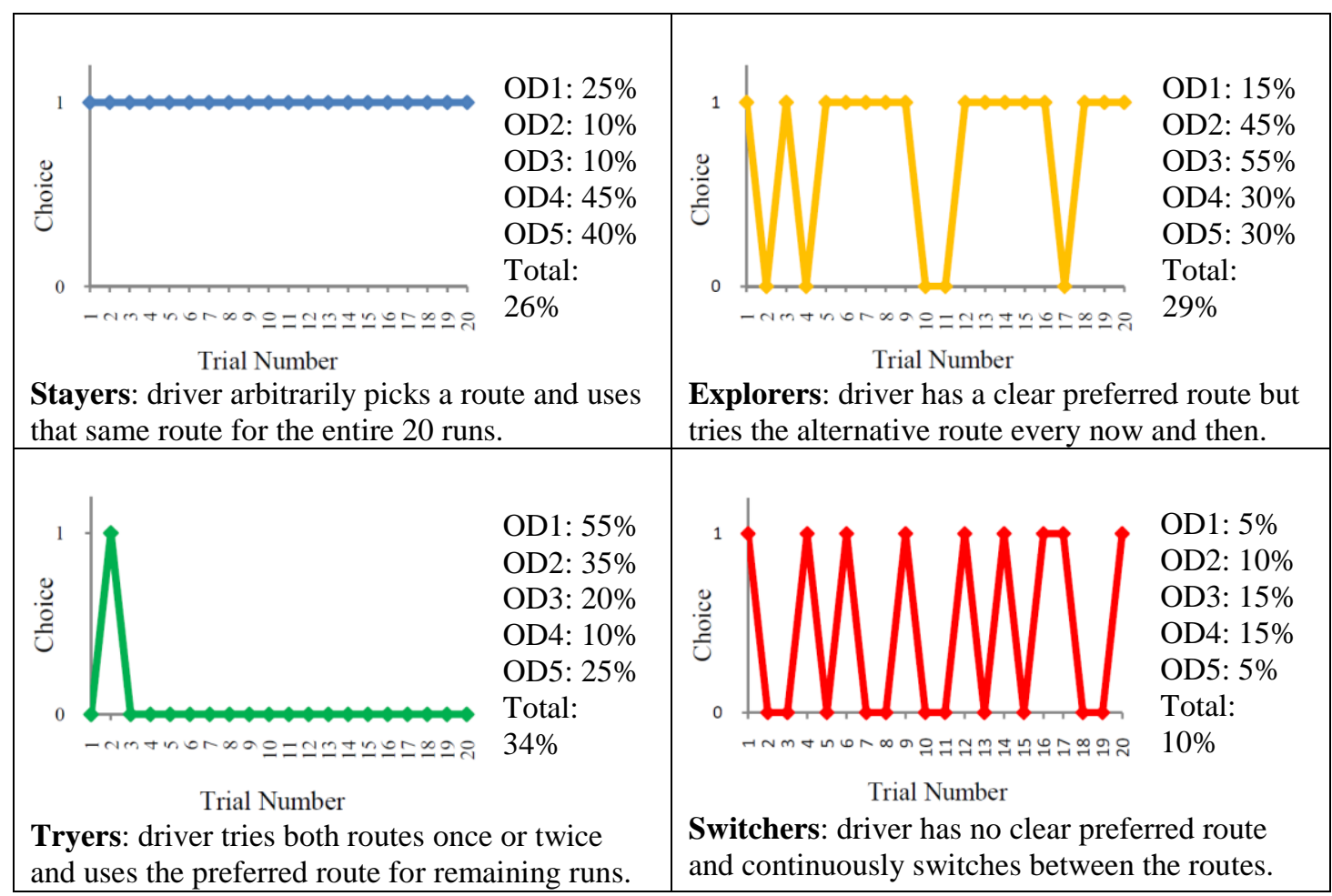

Figure 3. Driver Behaviour Types 
Note that some drivers behaved differently for different OD-pairs. For example, a driver could be a Switcher for OD-pair 1, but a Stayer for OD-pair 4. Overall, a majority of the participants had a clear preference for one of the routes and almost never switched (i.e., Stayers or Tryers). These behaviours occur more on OD-pairs with distinct routes such as OD-pairs 4 and 5. There are more Explorers on OD-pairs with routes that are similar, which is the case for OD-pair 1. Lastly, Switchers are mostly observed on OD-pairs 3 and 4, which are cases in which the shortest time route suffers from dense traffic conditions and unreliable travel times.

Now exploring route switching propensity, the data shows that participants on average switched in $20 \%$ of the cases. The number of switches was slightly above average for ODpairs 1 and 2, and slightly below average on OD-pairs 4 and 5. Remarkably, for OD-pair 4 this is in contrast to the relatively large share of Switchers which might be explained by the risk being caught in campus traffic and the relatively large share of Stayers.

Figure 4 shows that the number of route switches decreased with the number of runs. Hence, the number of switches in the first 10 runs is significantly higher than in the last 10 runs. This shift from explorative choice behaviour to exploiting choice behaviour has been observed by others [e.g., 29] and is a sign of learning and convergence. Moreover, in an earlier work, Tawfik and Rakha [24] showed that the percentage of participants choosing a route seems to converge. However, they found that only on OD-pairs 3 and 5 these percentages converged in line with the stochastic user equilibrium expectations. Again this shows that a simple model using travel time alone does not explain the data.

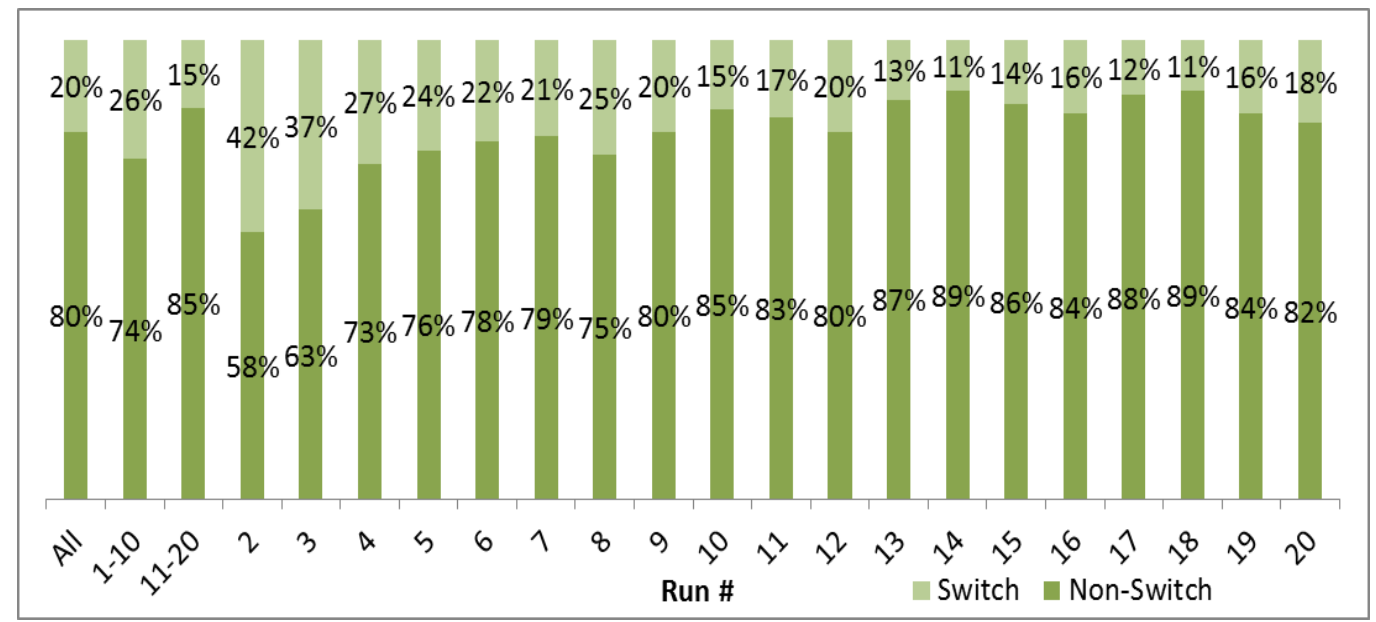

Figure 4. Evolution of Route Switching

\subsection{Choice Strategies}

In a normal route choice situation it is usually assumed that drivers compare the expected travel time of the available choice alternatives. Alternatively, in case of satisficing or inertia it is assumed that the driver only compares the last experienced travel time of the current choice with the expected travel time of that choice. Based on the available choice data we will evaluate both perspectives by calculating the expected saving and experienced saving respectively. Considering that these savings are either a gain or a loss, and the decision of the driver is to switch routes or repeat the current choice, four choice strategies can be distinguished:

- Choice strategy 1 (CS1): Gain and stay - the current choice performs better than expected and/or better than the choice alternative. The driver repeats the current choice at $\mathrm{t}+1$. This behaviour resembles travel time minimizing behaviour. 
- Choice strategy 2 (CS2): Loss and stay - the current choice performs worse than expected and/or worse than the choice alternative. Yet, the driver repeats the current choice at $\mathrm{t}+1$. This behaviour inertial behaviour.

- Choice strategy 3 (CS3): Gain and switch - the current choice performs better than expected and/or better than the choice alternative. Yet, the driver switches to the choice alternative $t+1$. This behaviour resembles compromising behaviour, possibly due to other factors than time.

- Choice strategy 4 (CS4): Loss and switch - the current choice performs worse than expected and/or worse than the choice alternative. The driver switches to the choice alternative at $\mathrm{t}+1$. This behaviour resembles rational travel time minimizing behaviour.

from the perspective of expected savings (compare choice alternatives), run 6 is an example of CS4 because gain was expected based on the average experienced travel times after run 5. Conversely, run 13 is an example of CS3 as loss was expected but the driver switched nonetheless. Run 11 is an example of CS2 and runs 16 to 20 are examples of CS1. From the perspective of experienced savings (compare current route), run 6 is an example of CS3 because gain was experienced in the previous run, but the driver switched. Run 9 is an example of CS2 because loss was experienced but the driver did not switch. Finally, run 17 is an example of CS1 and run 10 is an example of CS4.

\section{Results from the Perspective of Expected Savings}

\subsection{Choice Behaviour}

To reiterate, expected savings are based on the average experienced travel times of a participant on both routes. Table 2 gives an overview of the frequencies of the choice strategies based on expected savings. The sum of CS1 and CS4 shows that the majority of the choices were travel time minimising choices. Differences between OD-pairs and routes can largely be explained by the average travel time differences between the routes alternatives. For example, the correlation coefficients of CS1 and CS4 with the average travel time difference between routes imply that larger differences in travel time increase the frequency of these 'rational' strategies. This finding is intuitive. Similarly, the frequency of CS3 decreases when the travel time difference increases. Conversely, the frequency of CS2, i.e. inertial choices cannot be explained by travel time as is indicated by the correlation coefficient. Nevertheless, the data suggests that CS2 was highest for OD-pairs with small travel time differences or when the non-shortest time route is competitive with another route attribute like average speed (e.g., routes 2 and 4 ).

Table 2. Choice Strategies by OD-pair based on Expected Savings

\begin{tabular}{|c|c|c|c|c|c|c|c|}
\hline \multirow{2}{*}{$\begin{array}{l}\text { OD- } \\
\text { Pair }\end{array}$} & \multirow{2}{*}{ Route } & \multirow{2}{*}{ TT } & \multirow{2}{*}{$\begin{array}{l}\text { Avg. } \\
\Delta \text { STT }\end{array}$} & CS1 & CS2 & CS3 & CS4 \\
\cline { 5 - 8 } & & & & Gain & Loss & Gain & Loss \\
\hline \multirow{2}{*}{1} & 1 & 8.5 & 0.1 & $7 \%$ & $3 \%$ & $9 \%$ & $7 \%$ \\
\cline { 3 - 8 } & 2 & 8.4 & -0.1 & $33 \%$ & $26 \%$ & $6 \%$ & $9 \%$ \\
\hline \multirow{2}{*}{2} & 3 & 15.2 & -1.5 & $38 \%$ & $15 \%$ & $9 \%$ & $2 \%$ \\
\cline { 2 - 8 } & 4 & 16.7 & 1.5 & $5 \%$ & $20 \%$ & $2 \%$ & $10 \%$ \\
\hline \multirow{2}{*}{3} & 5 & 7.7 & -1.6 & $62 \%$ & $4 \%$ & $10 \%$ & $1 \%$ \\
\hline \multirow{2}{*}{4} & 6 & 9.3 & 1.6 & $0 \%$ & $11 \%$ & $1 \%$ & $11 \%$ \\
\hline & 7 & 10.2 & 0.6 & $2 \%$ & $7 \%$ & $4 \%$ & $11 \%$ \\
\hline
\end{tabular}




\begin{tabular}{|c|c|c|c|c|c|c|c|}
\hline \multirow{2}{*}{5} & 9 & 10.5 & 2.5 & $0 \%$ & $4 \%$ & $1 \%$ & $12 \%$ \\
\hline & 10 & 8.0 & -2.5 & $74 \%$ & $1 \%$ & $9 \%$ & $1 \%$ \\
\hline \multicolumn{4}{|c|}{ Total } & $51 \%$ & $24 \%$ & $12 \%$ & $13 \%$ \\
\hline \multicolumn{4}{|c|}{ Correl. $\Delta \mathrm{TT}$} & -0.92 & 0.10 & -0.89 & 0.93 \\
\hline \multicolumn{4}{|c|}{ DBT2: Tryers } & $66 \%$ & $27 \%$ & $3 \%$ & $4 \%$ \\
\hline \multicolumn{4}{|c|}{ DBT3: Explorers } & $41 \%$ & $22 \%$ & $18 \%$ & $19 \%$ \\
\hline \multicolumn{4}{|c|}{ DBT4: Switchers } & $27 \%$ & $19 \%$ & $27 \%$ & $27 \%$ \\
\hline
\end{tabular}

On an aggregated level the data reveals that if gain was expected from repeating the current choice (CS1+CS3: 63\%), 81\% of the participants made a rational choice and stayed at the current choice while 19\% decided to switch. In case loss was expected (CS2+CS4: $37 \%$ ), $65 \%$ made an inertial choice by staying at the current choice while $35 \%$ switched. From a behavioural perspective, if participants did not switch (CS1+CS2: 75\%) this can be explained by the expected saving in $68 \%$ of the cases. Similarly, switching (CS3+CS4: 25\%) was 'rational' in $52 \%$ of the cases. The relatively low frequency of switching and rational choices suggest that the participants were switch-averse and not necessarily interested in pursuing travel time gain, but more interested in avoiding the risk and travel time loss. As a result, a considerable share of the choices cannot be explained by travel minimisation alone.

Table 2 also shows the frequency of the choice strategies for the driver behaviour types Tryers, Explorers and Switchers. Note that Stayers lack the precondition of at least one experience of both choice alternatives. The figures show that the frequency of rational choices (i.e. CS1 and CS4) was highest for Tryers and lowest for Switchers. As Switchers switched the most and Tryers switched the least, this implies that more switching was not necessarily related to more expected gain. Moreover, for Switchers the frequencies of the choice strategies are in the same range which underlines the random character of this Driver Behaviour Type. Average travel times per driver behaviour type in Table 3 confirms that Switchers performed the worst as their experienced travel times were largest, while Stayers performed by far the best as their experienced travel times were smallest. The most logical explanation for this finding is that the participants had prior knowledge of the choice situations.

Table 3. Average Travel Times per Driver Behaviour Type

\begin{tabular}{|c|c|c|c|c|c|c|}
\hline $\begin{array}{c}\text { Average TT } \\
\text { [95\% conf. int.] }\end{array}$ & $\begin{array}{c}\text { OD-pair } \\
\mathbf{1}\end{array}$ & $\begin{array}{c}\text { OD-pair } \\
\mathbf{2}\end{array}$ & $\begin{array}{c}\text { OD-pair } \\
\mathbf{3}\end{array}$ & $\begin{array}{c}\text { OD-pair } \\
\mathbf{4}\end{array}$ & $\begin{array}{c}\text { OD-pair } \\
\mathbf{5}\end{array}$ & SUM \\
\hline \multirow{2}{*}{ Stayers } & 8.14 & 15.79 & 8.95 & 9.56 & 8.05 & 9.25 \\
& {$[0.24]$} & {$[0.27]$} & {$[0.40]$} & {$[0.15]$} & {$[0.16]$} & {$[0.10]$} \\
\hline \multirow{2}{*}{ Tryers } & 9.91 & 15.59 & 7.97 & 9.87 & 8.14 & 10.45 \\
& {$[0.39]$} & {$[0.23]$} & {$[0.16]$} & {$[0.19]$} & {$[0.19]$} & {$[0.10]$} \\
\hline \multirow{2}{*}{ Explorers } & 8.27 & 15.91 & 8.32 & 9.79 & 8.37 & 10.24 \\
& {$[0.20]$} & {$[0.28]$} & {$[0.24]$} & {$[0.38]$} & {$[0.30]$} & {$[0.12]$} \\
\hline \multirow{2}{*}{ Switchers } & 8.66 & 16.23 & 8.53 & 9.85 & 10.44 & 10.67 \\
& {$[0.50]$} & {$[0.37]$} & {$[0.29]$} & {$[0.26]$} & {$[0.52]$} & {$[0.16]$} \\
\hline
\end{tabular}

Next to the frequency of the four choice strategies we next examine how these evolve relative to the expected saving in case of switching (see Figure 5). It clearly shows that with increasing expected savings all behaviour becomes rational (i.e., CS1 and CS4). However, for both gains and losses smaller than approximately 1.5 minutes there is no clear difference between the saving intervals. This suggests that within this range all expected gains or losses have a similar effect on choice behaviour. Presumably this indicates that drivers' ability to correctly judge situations that involve small travel time differences is limited which is in line with literature. Moreover, inertial behaviour (CS2) occurs more with smaller gains. 


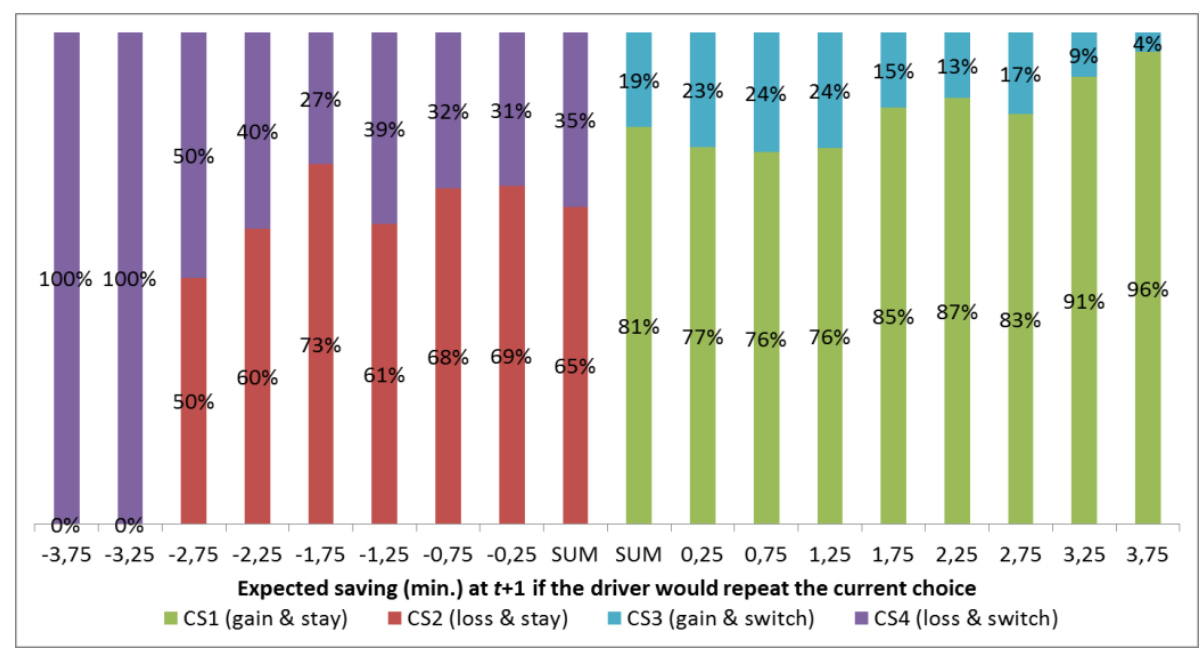

Figure 5. Frequency of Choice Strategies by Expected Saving (min.)

\subsection{Probability of Switching}

To determine the switching probability, the number of switches was divided by the total number of decisions, for each saving interval. The result is shown in Figure 6. The average switching probability was $25 \%$ while the switching probability increases if the expected saving decreases and/or becomes negative. This finding is intuitive. Notably, the $95 \%$ confidence interval indicates that expected loss up to 2.5 minutes does not lead to significantly more switching than average. This bandwidth is an indicator for the inertial threshold. Another observation from the Figure is the asymmetry between expected gains and expected losses which suggest that participants were more sensitive to expected loss when staying than to expected gain when switching. This is in line with earlier findings and supported by theories of loss aversion [e.g., 30].

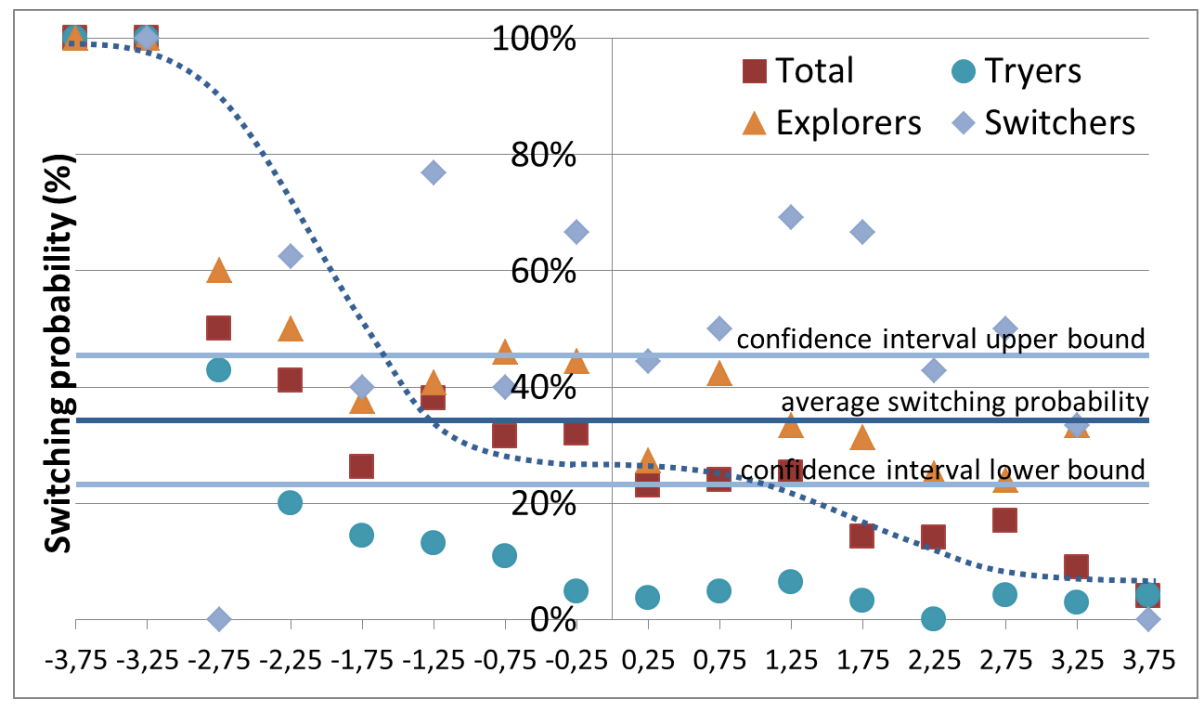

Figure 6. Switching Probability by Expected Saving (min.)

Figure 6 also shows the switching probability for Stayers, Explorers and Switchers (Stayers were excluded because they never switched). Note that the series for the Tryers reflects the one-sample experiences of the choice alternative that these drivers had. It shows that if the experienced saving on the choice alternative was a loss (i.e., the expected saving of switching back a gain), the participants were increasingly likely to switch back. Explorers and Switchers were much more likely to switch in general as defined by their type. However, 
Explorers show an intuitive pattern similar to the overall pattern, while the switching probability of Switchers does not seem to reveal any particular pattern. Overall, the results so far suggest that the choice strategies as applied by the participants were not necessarily bad, but could perhaps be applied in a more effective manner.

Finally, we analyse the lost travel time resulting from inertial choices as an indicator for the inertia threshold. Table 4 shows lost travel time statistics for each route for choice strategy 2 and cumulative statics for all choice strategies. The numbers represent the median and maximum expected saving, e.g. loss in case of CS2, in minutes and in percent. The Table shows that inertia thresholds up to 2.90 minutes and $33 \%$ of the average travel time occur. The median values indicate thresholds up to 1.22 minutes and $13 \%$ of the actual travel time. This order of magnitude is in line with figures reported in literature [e.g., 31, 32, 33]. However, there are considerable differences between OD-pairs and routes. Yet, there is no strong relation between the thresholds and the average travel time or the average travel time differences. This means that other factors, observed and non-observed, also affect thresholds. An important implication from these findings is that no generalised threshold exists, either in minutes or in percentage, but presumably that thresholds are dependent on the choice set and on route characteristics. Finally, Table 5 shows that losses involved in CS4 were generally larger than those of CS2. This suggests that larger loss induces more switching and that switching requires a minimum expected gain in return. The latter is another sign of inertial behaviour and thresholds and in line with literature.

Table 4. Lost Travel Time Statistics Expected Saving

\begin{tabular}{|c|c|c|c|c|c|c|}
\hline \multirow{2}{*}{$\begin{array}{c}\text { OD- } \\
\text { Pair }\end{array}$} & \multirow{2}{*}{ Route } & \multirow{2}{*}{$\begin{array}{c}\text { Avg. } \\
\text { TT }\end{array}$} & \multicolumn{2}{|c|}{ CS2 } & \multicolumn{2}{c|}{ CS2 } \\
\cline { 3 - 7 } & & & Median & Max. & Median & Max. \\
\hline \multirow{2}{*}{1} & 1 & 8.5 & -0.46 & -2.42 & $-5 \%$ & $-28 \%$ \\
\cline { 2 - 7 } & 2 & 8.4 & -0.44 & -2.78 & $-5 \%$ & $-33 \%$ \\
\hline \multirow{2}{*}{2} & 3 & 15.2 & -0.69 & -1.35 & $-5 \%$ & $-9 \%$ \\
\cline { 2 - 7 } & 4 & 16.7 & -0.99 & -2.74 & $-6 \%$ & $-16 \%$ \\
\hline \multirow{2}{*}{3} & 5 & 7.7 & -0.05 & -0.42 & $-1 \%$ & $-5 \%$ \\
\cline { 2 - 7 } & 6 & 9.3 & -1.22 & -2.65 & $-13 \%$ & $-28 \%$ \\
\hline \multirow{2}{*}{4} & 7 & 10.2 & -0.44 & -0.77 & $-4 \%$ & $-8 \%$ \\
\cline { 2 - 7 } & 8 & 9.6 & -1.08 & -2.90 & $-11 \%$ & $-30 \%$ \\
\hline \multirow{2}{*}{5} & 9 & 10.5 & -0.95 & -2.58 & $-9 \%$ & $-25 \%$ \\
\cline { 2 - 7 } & 10 & 8.0 & -0.78 & -0.78 & $-10 \%$ & $-10 \%$ \\
\hline \multicolumn{2}{|c|}{ CS2 (loss \& stay) } & -0.71 & -1.94 & $-7 \%$ & $-19 \%$ \\
\hline \multicolumn{2}{|c|}{ CS1 (gain \& stay) } & 0.81 & 3.29 & $8 \%$ & $36 \%$ \\
\hline \multicolumn{2}{l}{ CS4 (loss \& switch) } & -0.85 & -2.56 & $-8 \%$ & $-25 \%$ \\
\hline \multicolumn{2}{l}{ CS3 (gain \& switch) } & 0.90 & 2.91 & $10 \%$ & $30 \%$ \\
\hline
\end{tabular}

\section{Results from the Perspective of Experienced Savings}

\subsection{Choice Behaviour}

To reiterate, experienced savings are based on the average experienced travel time of the current route versus the last experienced travel time on that route. Table 5 gives an overview of the frequency of the choice strategies based on experienced savings. It shows that the frequency of CS2 (i.e., inertial behaviour) is higher for all OD-pairs compared to Table 2. This suggests that the participants were less responsive to experienced loss on the current route as opposed to expected loss relative to the choice alternative. 
The correlation coefficients indicate that the frequency of staying (i.e., CS1 and CS2) increases when the average travel time difference becomes more negative. Consequently, the frequency of these choice strategies is highest for shortest time routes. Compared to Table 2 the correlation coefficient for CS2 has a different sign and is statistically significant. The opposite is true for CS3 and CS4. This is another indication that choices were more affected by expected savings relative to the choice alternative than to experienced savings on to the current route.

On an aggregated level the data reveals that if gain was experienced (CS1+CS3: 57\%), 82\% of the participants made a rational choice and stayed at the current choice while $18 \%$ decided to switch. In case of experienced loss (CS2+CS4: 44\%), 82\% of the participants made an inertial choice by staying with the current choice while $18 \%$ switched. From a behavioural perspective, if participants did not switch (CS1+CS2: 83\%) this was an inertial choice in $44 \%$ of the cases. Similarly, switching (CS3+CS4: 18\%) cannot be explained by the experienced saving in 56\% of the cases). Table 5 also shows the frequency of the choice strategies for the four driver behaviour types. The figures show that all Driver Behaviour Types apart from the Switchers made more rational choices (i.e. CS1 and CS4) than other choices. Additionally, a counter-intuitive finding is that it seems that participants were a little more likely to switch after experiencing gain than after experiencing loss.

Table 5. Choice Strategies by OD-pair based on Experienced Savings

\begin{tabular}{|c|c|c|c|c|c|c|c|}
\hline \multirow{3}{*}{$\begin{array}{l}\text { OD- } \\
\text { Pair }\end{array}$} & \multirow{3}{*}{ Route } & \multirow{3}{*}{ TT } & \multirow{3}{*}{$\begin{array}{l}\text { Avg. } \\
\Delta \text { TT }\end{array}$} & CS1 & CS2 & CS3 & CS4 \\
\hline & & & & Gain & Loss & Gain & Loss \\
\hline & & & & Stay & Stay & Switch & Switch \\
\hline \multirow{2}{*}{1} & 1 & 8.5 & 0.1 & $12 \%$ & $9 \%$ & $5 \%$ & $7 \%$ \\
\hline & 2 & 8.4 & -0.1 & $34 \%$ & $24 \%$ & $4 \%$ & $6 \%$ \\
\hline \multirow[b]{2}{*}{2} & 3 & 15.2 & -1.5 & $32 \%$ & $23 \%$ & $4 \%$ & $6 \%$ \\
\hline & 4 & 16.7 & 1.5 & $14 \%$ & $12 \%$ & $5 \%$ & $4 \%$ \\
\hline \multirow{2}{*}{3} & 5 & 7.7 & -1.6 & $33 \%$ & $28 \%$ & $4 \%$ & $5 \%$ \\
\hline & 6 & 9.3 & 1.6 & $14 \%$ & $7 \%$ & $5 \%$ & $4 \%$ \\
\hline \multirow{2}{*}{4} & 7 & 10.2 & 0.6 & $7 \%$ & $3 \%$ & $3 \%$ & $4 \%$ \\
\hline & 8 & 9.6 & -0.6 & $41 \%$ & $34 \%$ & $4 \%$ & $4 \%$ \\
\hline & 9 & 10.5 & 2.5 & $1 \%$ & $1 \%$ & $3 \%$ & $3 \%$ \\
\hline & 10 & 8.0 & -2.5 & $46 \%$ & $40 \%$ & $2 \%$ & $5 \%$ \\
\hline \multicolumn{4}{|c|}{ Total } & $47 \%$ & $36 \%$ & $10 \%$ & $8 \%$ \\
\hline \multicolumn{4}{|c|}{ Correl. $\Delta \mathrm{TT}$} & -0.87 & -0.87 & 0.32 & -0.43 \\
\hline \multicolumn{4}{|c|}{ DBT1: Stayers } & $54 \%$ & $46 \%$ & $\mathrm{n} / \mathrm{a}$ & $\mathrm{n} / \mathrm{a}$ \\
\hline \multicolumn{4}{|c|}{ DBT2: Tryers } & $54 \%$ & $40 \%$ & $4 \%$ & $2 \%$ \\
\hline \multicolumn{4}{|c|}{ DBT3: Explorers } & $40 \%$ & $26 \%$ & $18 \%$ & $16 \%$ \\
\hline \multicolumn{4}{|c|}{ DBT4: Switchers } & $24 \%$ & $21 \%$ & $30 \%$ & $24 \%$ \\
\hline
\end{tabular}

Next to the frequency of the four choice strategies we next examine how these evolve relative to the experienced saving (see Figure 5). It clearly shows that with increasing experienced gains all behaviour eventually becomes rational (i.e., CS1). No particular pattern can be observed for experienced loss which is a sign that factors other than the experienced loss led to switching. Finally, the Figure shows that for losses up to 2 minutes there is no clear difference between the intervals. This is an indication of similar behaviour within this range which might be explained by driver' limited ability to correctly judge (or care about) situations that involve small travel time differences. As a consequence, inertial behaviour (CS2) occurs more with smaller gains. 


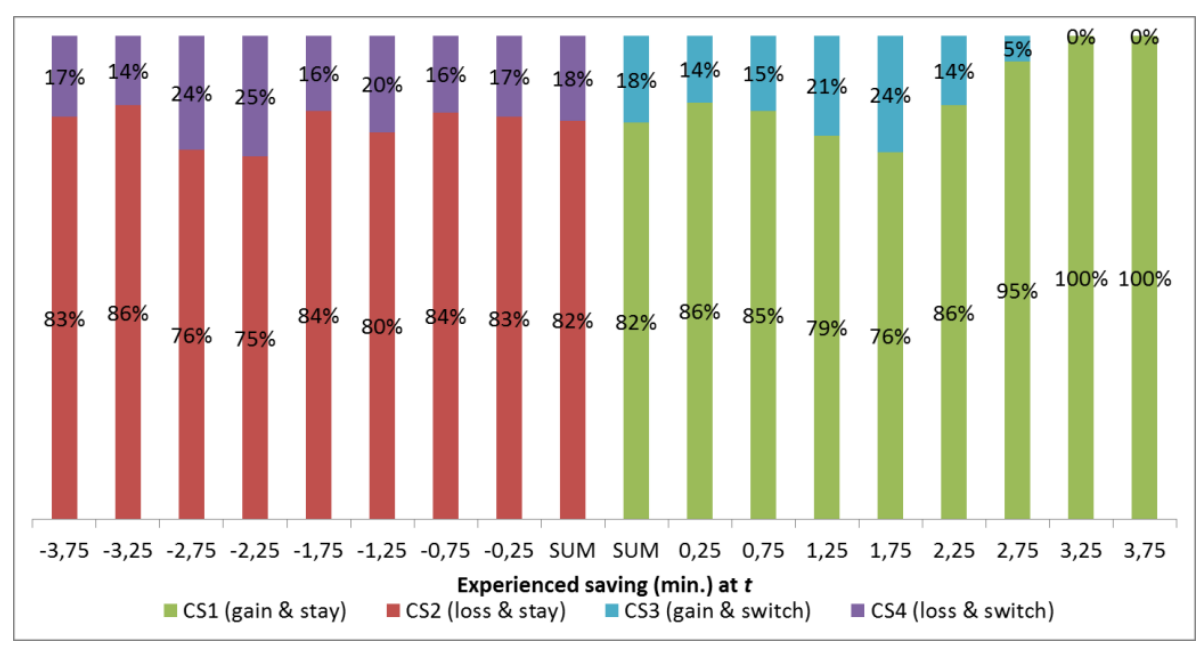

Figure 7. Frequency of Choice Strategies by Experienced Saving (min.)

\subsection{Probability of Switching}

To determine the switching probability, the number of switches was divided by the total number of decision, for each saving interval. The result is shown in Figure 8. The average switching probability was $20 \%$ but the Figure does not show a clear pattern in relation to the experienced saving as most values are within the $95 \%$ confidence interval. The absence of a clear pattern suggests that the experienced saving alone is not a major driver for route switching. One explanation might be inertial behaviour, another that the experienced saving is likely to be considered cojointly with the availability of choice alternatives and the expected saving of these. Figure 8 also shows the switching probability for Tryers, Explorers and Switchers (Stayers were excluded because they never switched). Again it is difficult to derive a pattern although Explorers seem increasingly likely to switch with larger losses.

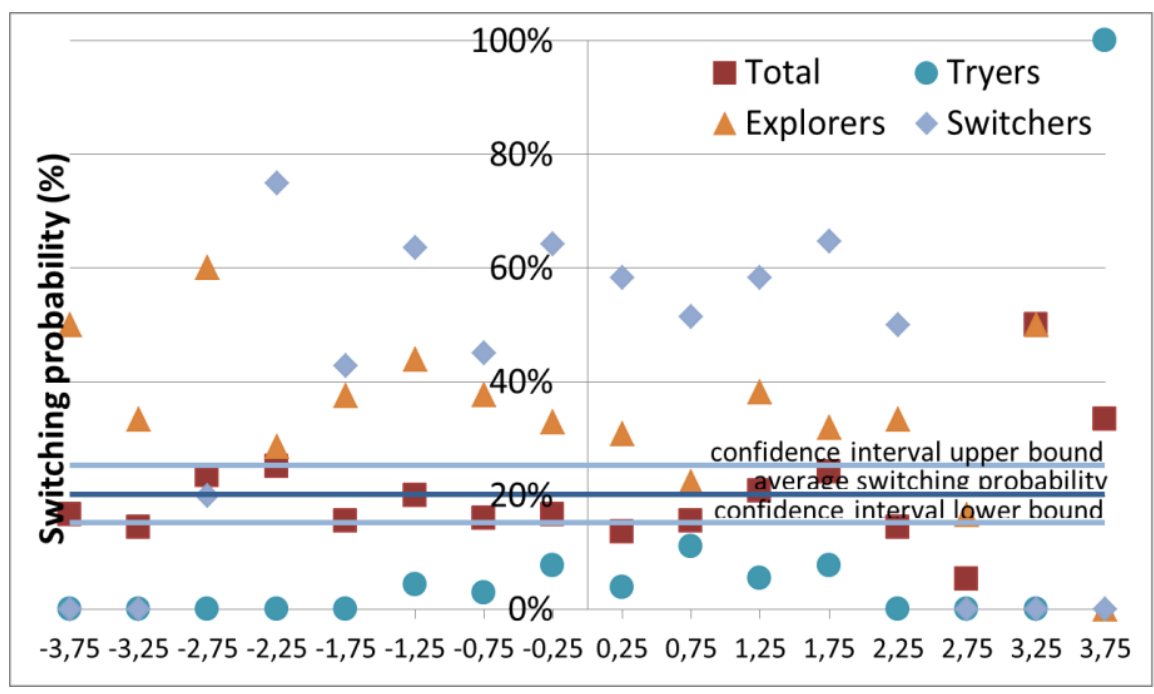

Figure 8. Switching Probability by Experienced Saving (min.)

Finally, we analyse the lost travel time resulting from inertial choices as an indicator for the inertia threshold. Error! Not a valid bookmark self-reference. shows lost travel time statistics for each route for choice strategy 2 and cumulative statics for all choice strategies. The figures represent the median and maximum experienced saving, e.g., loss in case of CS2, in minutes and in percent. The Table shows that inertia thresholds up to 3.77 minutes 
and $47 \%$ of the average travel time occur. The median values indicate thresholds up to 0.97 minutes and $11 \%$ of the average travel time. Compared to Table 4 the thresholds seem more systematic and repetitive although difference between OD-pairs and routes remain. Generally, thresholds for shortest time routes appear to be larger but this becomes less apparent when other factors than time are at play which is the case for OD-pair 2 and 3. To confirm an earlier finding; values for thresholds cannot be generalised as the data suggests that they are dependent on the choice set and on the route characteristics.

Table 6. Lost Travel Time Statistics Experienced Saving

\begin{tabular}{|c|c|c|c|c|c|c|}
\hline \multirow{2}{*}{ OD-Pair } & \multirow{2}{*}{ Route } & \multirow{2}{*}{ Avg.TT } & \multicolumn{2}{|c|}{ CS2 } & \multicolumn{2}{c|}{ CS2 } \\
\cline { 3 - 7 } & & & Median & Max. & Median & Max. \\
\hline \multirow{2}{*}{1} & 1 & 8.5 & -0.97 & -3.20 & $-11 \%$ & $-38 \%$ \\
\cline { 2 - 6 } & 2 & 8.4 & -0.76 & -3.72 & $-9 \%$ & $-44 \%$ \\
\hline \multirow{2}{*}{2} & 3 & 15.2 & -0.67 & -2.75 & $-4 \%$ & $-18 \%$ \\
\cline { 2 - 7 } & 4 & 16.7 & -0.62 & -3.51 & $-4 \%$ & $-21 \%$ \\
\hline \multirow{2}{*}{3} & 5 & 7.7 & -0.56 & -2.25 & $-7 \%$ & $-29 \%$ \\
\cline { 2 - 7 } & 6 & 9.3 & -0.51 & -3.02 & $-5 \%$ & $-32 \%$ \\
\hline \multirow{2}{*}{4} & 7 & 10.2 & -0.80 & -2.87 & $-8 \%$ & $-28 \%$ \\
\cline { 2 - 7 } & 8 & 9.6 & -0.70 & -3.58 & $-7 \%$ & $-37 \%$ \\
\hline \multirow{2}{*}{5} & 9 & 10.5 & -0.21 & -0.77 & $-2 \%$ & $-7 \%$ \\
\cline { 2 - 7 } & 10 & 8.0 & -0.59 & -3.77 & $-7 \%$ & $-47 \%$ \\
\hline \multicolumn{2}{|c|}{ CS2 (loss \& stay) } & -0.64 & -3.77 & $-7 \%$ & $-30 \%$ \\
\hline \multicolumn{2}{|c|}{ CS1 (gain \& stay) } & 0.73 & 2.78 & $8 \%$ & $32 \%$ \\
\hline \multicolumn{2}{|c|}{ CS4 (loss \& switch) } & -0.78 & -4.52 & $-8 \%$ & $-29 \%$ \\
\hline \multicolumn{2}{|c|}{ CS3 (gain \& switch) } & 0.82 & 4.13 & $9 \%$ & $30 \%$ \\
\hline
\end{tabular}

\section{Conclusion}

\subsection{Approach}

Inertial choice behaviour shows that drivers make choices that are satisfactory rather than optimal. Consequently, drivers may not necessarily respond to changes in the traffic system. In this paper, route choice data from a real-world driving experiment was used to study inertial behaviour. It aimed to observe the frequency of different choice strategies, examine route switching behaviour and inertial choices in particular, and to estimate inertia thresholds. For the analysis, average experienced travel times and expected travel times were used to calculate expected savings relative to choice alternatives and experienced savings on the current route.

\subsection{Main Findings}

With regards to the research questions that were defined in the beginning of this paper the main findings are the following: (1) on average about 1/4th of the choices concerned inertial behaviour as based on expected savings. When two alternatives have similar travel times or when non-travel time attributes are dominant, the amount of inertial choices increases. Based on experienced savings, a little more than one-third of the choices concerned inertial behaviour on average. Compared to results from expected savings this suggests that the participants were less responsive to experienced loss on the current route as opposed to expected loss relative to the choice alternative; (2) for expected savings and based on lost travel time for routes with an average travel time of 8 to 16 minutes, inertia thresholds up to 
2.90 minutes or $33 \%$ of the average actual travel time and a median of 1.22 minutes or $13 \%$ of the average actual travel time were found. For experienced savings, inertia thresholds up to 3.77 minutes or $47 \%$ of the average actual travel time and a median of 0.97 minutes or $11 \%$ of the average actual travel time were found. As differences between routes were considerable, it appears that the behavioural principles are general and systematic, but that the magnitude is probabilistic and dependent on the choice set; (3) as loss related to switching was on average larger than loss related to repeating the current choice, switching seems to require a minimum expected loss. This is another sign of inertial behaviour and thresholds; (4) for expected and experienced loss up to 1.5-2 minutes there was no clear difference in the frequency of choice strategies. This might be explained by driver' limited ability to correctly judge (or care about) situations that involve small travel time differences; (5) four driver behaviour types and four choice strategies were distinguished showing that the majority of participants hardly ever switched to adopt a travel time minimising strategy. Nonetheless, for a considerable share of choices a simple model using travel time alone does not explain the data; (6) additionally, it was found that the travel time difference between alternatives, average travel speed and travel time reliability influence choice strategies next to travel time, and (7) asymmetry between gains and losses was found in several ways. In general it appeared that respondents were loss-averse rather than gain-seeking, which made them switch-averse.

When applied in the context of transport, inertial behaviour and related thresholds may give policy makers, road operators and traffic engineers an incentive 'lever' to modify choice attributes, which in turn will increase the performance of the traffic system. The aforementioned findings offer an illustration of the size of thresholds under different circumstances. Examples of their application are given in $[9,10]$ which showed a reduction in total travel time of 5\% compared to user equilibrium in case of an threshold of 4 minutes for a trip of approximately 22 minutes. Additionally, the improvement in the network performance increased with an increasing threholds, up to $14 \%$ in case of a threshold of 10 minutes.

\subsection{Future Research}

Several improvements can be made to the research presented in this paper: (1) switching thresholds were defined as a fixed value while in reality it is likely that they are subject to successive good and/or bad experiences. A moving average as proposed by Van Berkum and Van der Mede [13] might better capture drivers' sensitivity based on not only the current or last situation but also other recent ones; (2) as suggested by findings on switching probability, it is expected that inertia thresholds are different for choice situation and different driver behaviour types. Additionally, experienced travel times are not only the result of choices but also of stochasticity in the traffic system. To better understand the probabilistic nature of inertia thresholds these relations should be further explored; (3) it is worth to further explore the effects of the asymmetry between gains and losses and define a framework that integrates theories of loss aversion, indifference bands and driver heterogeneity in relation to switching probability; (4) traffic information is expected to affect drivers' perception and route choice behaviour. Therefore it is relevant to study how the presence of advanced traffic information systems affect the findings presented in this paper, and (5) lastly it is appropriate to estimate a model based on the results presented in this paper that better matches the data than a simple model that is based on travel time alone does [e.g., 34, 35, 36]. At the same time, expected savings relative to choice alternative and experienced savings on the current routes, and their effect on choice behaviour should be examined simultaneously as in the mind of the driver they are probably not independent from one another either. 


\section{Acknowledgments}

The real-world driving experiment was approved by the Virginia Tech Institutional Review Board. The authors would like to acknowledge the financial support from the Connected Vehicle Initiative University Transportation Centre (CVI-UTC). The work presented in this paper is part of a $\mathrm{PhD}$ research funded by Imtech Traffic \& Infra and the University of Twente (both in the Netherlands).

\section{References}

[1] E. Avineri and J. N. Prashker, "Violations of expected utility theory in route-choice stated preferences", Transportation Research Record, vol. 1894, (2004), pp. 222-229.

[2] H. A. Simon, "A behavioural model of rational choice," Quarterly Journal of Economics, vol. 69, (1955), pp. 99-118.

[3] D. Kahnemann, "Thinking, fast and slow", vol. First edition. New York: Farrar, Straus and Giroux, (2011).

[4] D. McFadden, "Rationality for Economists?", Journal of Risk and Uncertainty, vol. 19, (1999), pp. 73-105.

[5] H. S. Mahmassani and G.-L. Chang, "On boundedly rational user equilibrium in transportation networks", Transportation Science, vol. 21, pp. 89-99, (1987).

[6] V. Cantillo and J. Ortúzar, "Implications of thresholds in discrete choice modelling," Transport Reviews, vol. 26, (2006), pp. 667-691.

[7] T. Cram, "Smarter pricing: how to capture more value in your market", Harlow: Pearson Education Limited, (2006).

[8] D. Levy, H. Chen, S. Ray and M. Bergen, "Asymetric price adjustment in the smal: an implication of rational inattention," Tjalling C. Koopmans Research Institute, Utrecht School of Economics, pp. Discussion paper Serier nr: 04-23, (2004).

[9] J. Vreeswijk, J. Bie, E. Van Berkum, and B. Van Arem, "Effective traffic management based on bounded rationality and indifference bands," IET Intelligent Transport Systems, vol. 7, (2013a), pp. 265-274.

[10] J. D. Vreeswijk, R. L. Landman, E. C. Van Berkum, A. Hegyi, S. P. Hoogendoorn and B. Van Arem, "Improving the road network performance with dynamic route guidance by considering the indifference band of road users", presented at the Proceedings of the 13th International Conference on Travel Behaviour Research, Toronto, (2012b).

[11] B. Verplanken, H. Aarts and A. Van Knippenberg, "Habit, information acquisition, and the process of making travel mode choices", European Journal of Social Psychology, vol. 27, (1997), pp. 539-560.

[12] J. W. Payne, J. R. Bettman and E. J. Johnson, "The adaptive decision maker", Cambridge: Cambridge University Press, (1993).

[13] E. C. Van Berkum and P. H. J. Van der Mede, "The impact of traffic information", PhD-thesis, Delft University of Technology, Delft, (1993).

[14] C. Chorus and B. Dellaert, "Travel Choice Inertia - The Joint Role of Risk Aversion and Learning", Journal of Transport Economics and Policy, vol. 46, (2012), pp. 139-155.

[15] K. K. Srinivasan and H. S. Mahmassani, "Modeling intertia and compliance mechnisms in route choice behavior under real-time information", Transportation Research Record, vol. 1725, (2000), pp. 45-53.

[16] K. Monroe, "Buyers subjective perceptions of price", Journal of Marketing Reseach, vol. 10, pp. 70-80, (1970).

[17] C. G. Chorus and H. J. P. Timmermans, "Measuring user benefits of changes in the transport system when traveler awareness is limited", Transportation Research Part A: Policy and Practice, vol. 43, pp. 536-547, (2009).

[18] A. Tversky, "Elemination by aspects: a theory of choice", Psychological Review, vol. 79, (1972), pp. $291-$ 299.

[19] M. Ben-Akiva and B. Boccara, "Discrete choice models with latent choice sets," International Journal of Research in Marketing, vol. 12, (1995), pp. 9-24.

[20] C. G. Prato and S. Bekhor, "Modeling route choice behavior: How relevant is the composition of choice set?", Transportation Research Record, vol. 2003, (2007), pp. 66-73.

[21] J. Vreeswijk, T. Thomas, E. Van Berkum and B. Van Arem, "Perception bias in route choice", Transportation, vol. 41, (2014), pp. 1-17. Una

[22] J. Vreeswijk, T. Thomas, E. Van Berkum and B. Van Arem, "Drivers' perception of route alternatives as indicator for the indifference band", Transportation Research Record, vol. 2383, pp. 10-17, (2013c).

[23] A. Tawfik and H. Rakha, "A real-world route choice experiment to investigate and model driver perceptions," presented at the Proceedings of the 91st annual meeting of the Transportation Research Board, Washington D.C., (2012b)

[24] A. Tawfik and H. Rakha, "Network route-choice evoluation in real-life experiment: necessary shift from network- to driver-oriented modeling," Transportation Research Record, vol. 2322, (2012a).

[25] A. J. Horowitz, "The stability of stochastic equilibrium in a two-link transportation network," Transportation Research Part B: Methodological, vol. 18, pp. 13-28, (1984). 
[26] H. S. Mahmassani and K. K. Srinivasan, "Under real-time information: heuristics and adjustment processes," in Human behaviour and traffic networks, M. S. Schreckenberg, R., Ed., ed Berlin/Heidelberg/New York: Springer-Verlag, (1995).

[27] H. S. Mahmassani and G.-L. Chang, "Experiments with departure time choice dynamics of urban commuters", Transportation Research Part B: Methodological, vol. 20, (1986), pp. 297-320.

[28] M. Ben-Akiva, A. De Palma, and K. Isam, "Dynamic network models and driver information systems", Transportation Research Part A: Policy and Practice, vol. 25, (1991), pp. 251-266.

[29] P. Senk, "Route choice under microscope: Process oriented analysis of decision making," in Proceedings of the 89th annual meeting of the Transportation Research Board, Washington D.C., (2010).

[30] D. Kahnemann and A. Tversky, "Prospect theory: an analysis of decision under risk", Econometrica, vol. 47, (1979), pp. 263-291.

[31] K. K. Srinivasan and H. S. Mahmassani, "Role of congestion and information in trip-makers' dynamic decision processes experimental investigation", Transportation Research Record, (1999), pp. 44-52.

[32] H. S. Mahmassani and Y. H. Liu, "Dynamics of commuting decision behaviour under Advanced Traveller Information Systems", Transportation Research Part C: Emerging Technologies, vol. 7, pp. 91-107, (1999).

[33] H. S. Mahmassani and G.-L. Chang, "Dynamic aspects of departure-time choice behavior in a commuting system: theoretical framework and experimental analysis", Transportation Research Record, vol. 1037, pp. 88-101, (1985).

[34] L. Zhang, "Search, information, learning and knowledge in travel decision making - A positive approach for travel behavior and demand analysis", PhD-thesis, University of Minnesota, Twin Cities, (2006).

[35] C. Carrion, "Travel Time Perception Errors: Causes and Consequences", PhD-thesis, University of Minnesota, Minneapolis-St. Paul, United States, (2013).

[36] M. Van Essen, H. Rakha, J. Vreeswijk, L. Wismans and E. Van Berkum, "Day-to-day route choice modeling incorporating inertial behavior", Working paper, (2014). 